\title{
Beetroot juice consumption reduced blood pressure in normotensive individuals in an acute dose-response study
}

\author{
T. W. George, N. Kaffa and J. A. Lovegrove \\ Hugh Sinclair Unit of Human Nutrition, Department of Food and Nutritional Sciences and the Institute for Cardiovascular \\ and Metabolic Research (ICMR), The University of Reading, Whiteknights, PO Box 226, Reading, Berks RG6 6AP, UK
}

Elevated blood pressure (BP) is a leading preventable cause of morbidity and mortality and an established independent risk factor for CVD and stroke. Fruit and vegetable-rich diets have been shown to reduce systolic blood pressure (SBP) in hypertensive subjects ${ }^{(1,2)}$. Green leafy vegetables conferred the strongest protection ${ }^{(1)}$. These contain high concentrations of nitrate that can act as a substrate for the endogenous formation of the potent vasodilator nitric oxide after consumption ${ }^{(3)}$. Beetroot juice (BJ) is also rich in nitrate and has been shown to lower SBP by $10 \mathrm{mmHg}$ in normotensive individuals when given as a $500 \mathrm{ml}$ dose ${ }^{(4)}$.

The present study investigated the dose-response effects of BJ on $24 \mathrm{~h}$ ambulatory blood pressure (ABP). Twenty-five normotensive volunteers (18 males and 7 females), aged 18-64 years, consumed 3 doses of BJ (100, 250 and $500 \mathrm{ml})$ and a water/no BJ control in a single-blind, randomized and controlled crossover study. Low-nitrate mineral water was used to match the volume in all drinks to $500 \mathrm{ml}$. $\mathrm{ABP}$ was determined every $15 \mathrm{~min}$ for $1 \mathrm{~h}$ pre- and $4 \mathrm{~h}$ post-drink consumption, every $30 \mathrm{~min}$ for a further $9 \mathrm{~h}$ and hourly overnight. Urine was collected at $0,2,4,6$ and $24 \mathrm{~h}$ after BJ consumption. The subjects followed a low-nitrate diet for $48 \mathrm{~h}$ and consumed standardised low-nitrate meals the night before and during the study day.

SBP was significantly reduced $2-3 \mathrm{~h}$ post ingestion by $6.73,6.69$ and $9.82 \mathrm{mmHg}$ for 100,250 and $500 \mathrm{ml} \mathrm{BJ}$, respectively. Incremental area under the curve (IAUC) for SBP and diastolic BP (DBP) were significantly lower 0-240 min post ingestion $(P<0.001)$ after all BJ doses with SBP remaining lower up to $780 \mathrm{~min}(P<0.001)$. Urinary nitrate/nitrite was significantly higher than no BJ for all doses $(P<0.001)$ and remained significantly raised up to $24 \mathrm{~h}$ post consumption of $500 \mathrm{ml} \mathrm{BJ}(P=0.004)$. Urinary nitrate/nitrite IAUC was significantly and negatively correlated with IAUC for SBP and DBP $\left(r^{2}=-0.331, P=0.001\right.$ and $r^{2}=-0.240, P=0.017$, respectively). Urine antioxidant potential significantly increased after $500 \mathrm{ml} \mathrm{BJ}$ at $240 \mathrm{~min}$ post ingestion.

Overall, the study showed that BJ ingestion significantly, and in a near dose-dependent manner, lowered BP and increased urine nitrate/ nitrite levels in normotensive subjects. These findings support the existing evidence for a beneficial effect of nitrate-rich BJ in lowering $\mathrm{BP}$, which may contribute to the cardioprotective effects of vegetable-rich diets.

1. Joshipura KJ, Hu FB, Manson JE et al. (2001) The effect of fruit and vegetable intake on risk for coronary heart disease. Ann Intern Med 134, 11061114.

2. Sacks FM, Svetkey LP, Vollmer WM et al. (2001) Effects on blood pressure of reduced dietary sodium and the dietary approaches to stop hypertension (DASH) diet. $N$ Eng $J$ Med 344, 3-10.

3. Lundberg JO \& Govoni M (2004) Inorganic nitrate is a possible source for systemic generation of nitric oxide. Free Radic Biol Med 37, 395-400.

4. Webb AJ, Patel N, Loukogeorgakis S et al. (2008) Acute blood pressure lowering, vasoprotective, and antiplatelet properties of dietary nitrate via bioconversion to nitrite. Hypertension 51, 784-790. 